\title{
Aligned Magnetic Field Effects on Flow and Heat Transfer of the Upper-Convected Maxwell Fluid over a Stretching/Shrinking Sheet
}

\author{
Iskandar Waini ${ }^{1,{ }^{*}}$, Nurul Amira Zainal ${ }^{1}$, and Najiyah Safwa Khashi'ie $^{1}$ \\ ${ }^{1}$ Faculty of Engineering Technology, Universiti Teknikal Malaysia Melaka, Hang Tuah Jaya, 76100 \\ Durian Tunggal, Melaka, Malaysia
}

\begin{abstract}
In this paper, the effect of aligned magnetic field towards the flow and heat transfer of the upper-convected Maxwell (UCM) fluid over a stretching/shrinking sheet is numerically studied. The governing partial differential equations are reduced into a system of ordinary differential equations using a similarity transformation, which are then solved numerically using the shooting method. The skin friction and heat transfer coefficients, the velocity, as well as the temperature profiles of the fluid are presented and discussed. Results indicate that an increase in the aligned angle strengthens the applied magnetic field which decrease the velocity and increase the temperature profiles of the fluid. This implies that an increase in the aligned angle increases the skin friction coefficient and decreases the heat transfer coefficients.
\end{abstract}

\section{Introduction}

The fluid dynamics due to a stretching sheet are important in extrusion processes. The production of sheeting material arises in a number of industrial manufacturing processes and includes both metal and polymer sheets like the cooling of an infinite metallic plate in a cooling bath, paper production, glass blowing, etc. In view of these applications, Sakiadis [1] first investigated the boundary layer flow on a continuous solid surface moving at constant speed. Due to the entrainment of the ambient fluid, this boundary layer flow is quite different from the Blasius flow past a flat plate. Sakiadis's theoretical predictions for Newtonian fluids were later corroborated experimentally by Tsou et al. [2].

Since the pioneering study by Crane [3], who presented an exact analytical solution for the steady two-dimensional flow due to a stretching surface in a quiescent fluid, many authors have considered various aspects of this problem and obtained similarity solutions. Gupta and Gupta [4] investigated the heat and mass transfer corresponding to the similarity solutions for the boundary layer over a stretching sheet subjected to suction or blowing. Chen and Char [5] analyzed the heat transfer characteristics over a continuous stretching surface with variable surface temperature. Research involving the steady two-dimensional

*Corresponding author: iskandarwaini@utem.edu.my 
flow due to a stretching surface in a quiescent fluid, many authors has considered various aspects of this problem and obtained similarity solutions (see [6-9]).

Investigations of magnetohydrodynamic (MHD) flow are very important industrially and have applications in different areas of research such as petroleum production and metallurgical processes. The properties of the end product depend greatly on the rate of cooling involved in these processes and the desired properties of the end product can be controlled by the use of electrically conducting fluids and application of magnetic field. Besides, some electrically conducting fluids such as nuclear fuel slurries, liquid metals, mercury amalgams, biological fluids, lubrication oils and certain greases have many applications in the absence as well as in the presence of a magnetic field [10]. The problem involving the MHD boundary layer has been investigated by the number of authors (see [11-17]) by considering various effects.

However, some interest has been given to the study of non-Newtonian fluid which is viscoelastic fluid due to the numerous applications in the industry. Birds et al. [18] discussed that the viscoelastic fluid models used in previous works are simple models such as second-order model and/or Watler's B model which are known to be good only for weakly elastic fluids subject to slow and/or slowly-varying flows. Though, the models can describe the aforementioned problems adequately, but physically it is violating certain rules of thermodynamics. Furthermore, a non-Newtonian second grade fluid does not give meaning full results for highly elastic fluids (polymer melts) which occur at high Deborah numbers. Therefore, as far as polymer industry is concerned, the significance of the results reported in the above works is limited. Obviously, for the theoretical results to become of any industrial significance, more realistic viscoelastic fluid models such as upper-convected Maxwell model or Oldroyd-B model should be invoked in the analysis [19]. The boundary layer flow and heat transfer due to stretching sheet for the upper-convected Maxwell fluid has been considered by many researchers (see [20-29]) in various ways.

Recently, Raju et al. [30] have been reported the effects of aligned magnetic field on steady two-dimensional flow over a vertical stretching surface. They found that increase in aligned angle decreases the velocity profiles and increases the temperature and concentration profiles of the fluid. In addition, the effect of the aligned magnetic field and cross-diffusion on a nanofluid over an exponentially stretching surface in a porous medium has been studied by Sulochana et al. [31]. The researchers observed that an increase in the aligned angle decreases the velocity profiles and increases the temperature and concentration profiles of the nanofluid.

In this present study, we investigate the effect of aligned magnetic field towards the flow and heat transfer of the upper-convected Maxwell fluid over a stretching/shrinking sheet. The numerical results are tabulated and shown graphically to illustrate the influence of the various parameters on the velocity and temperature profiles, as well as on the skin friction and the heat transfer coefficients. As a contribution, this present study will gain a better understanding in terms of the fundamental scientific knowledge of the flow and heat transfer phenomena-related subjects. Besides, the theoretical predictions and numerical solutions obtained in this study will help to explain and verify experiments for the engineers.

\section{Mathematical formulation}

A steady two-dimensional boundary layer flow of an upper-convected Maxwell fluid over a stretching/shrinking sheet at $y=0$ is considered as shown in Figure 1. The stretching/shrinking velocity of the sheet is $u_{w}(x)=a x$, where $a$ is a positive constant 
$(a>0)$. Here, the aligned magnetic field $B_{0}$ is applied to the flow with acute angle $\gamma$ along $y$ direction. This magnetic field acts like a transverse magnetic field at $\gamma=\pi / 2$. The induced magnetic field due to the motion of the electrically conducting fluid is negligible. It is also assumed that the external electric field is zero and the electric field due to polarization of charges is negligible [13].

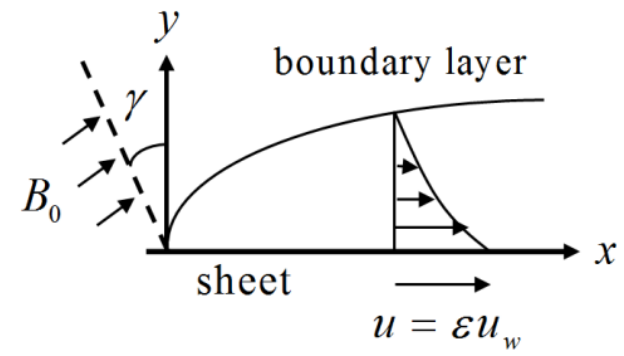

(a) Stretching case $(\varepsilon>0)$

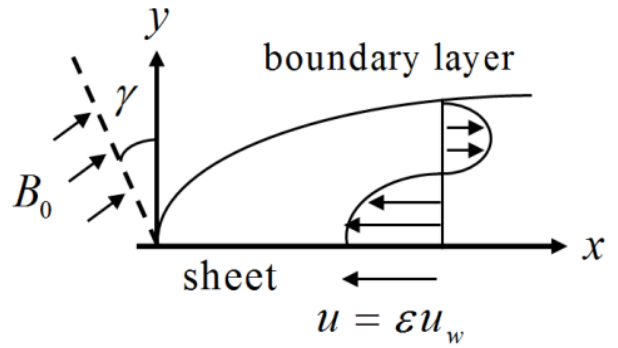

(b) Shrinking case $(\varepsilon<0)$

Fig. 1. Physical model and coordinate system.

By implementing the boundary layer approximations and applying the order of magnitude analysis, the governing equations for the upper-convected Maxwell fluid can be written as follows (see Abel et al. [19]):

$$
\begin{gathered}
\frac{\partial u}{\partial x}+\frac{\partial v}{\partial y}=0 \\
u \frac{\partial u}{\partial x}+v \frac{\partial u}{\partial y}+\lambda\left(u^{2} \frac{\partial^{2} u}{\partial x^{2}}+v^{2} \frac{\partial^{2} u}{\partial y^{2}}+2 u v \frac{\partial^{2} u}{\partial x \partial y}\right)=v \frac{\partial^{2} u}{\partial y^{2}}-\frac{\sigma B_{0}^{2}}{\rho} \sin ^{2}(\gamma) u \\
u \frac{\partial T}{\partial x}+v \frac{\partial T}{\partial y}=\frac{k}{\rho c_{p}} \frac{\partial^{2} T}{\partial y^{2}}
\end{gathered}
$$

where $u$ and $v$ are the velocity components along the $x$ and $y$ axes, respectively, and $T$ is the temperature of the fluid, $\rho$ is the fluid density, $\sigma$ is the electrical conductivity of the fluid and $\lambda$ is the relaxation time parameter of the fluid, while $v$ is the kinematic viscosity of the fluid, $c_{p}$ is the specific heat at a constant pressure, $k$ is the thermal conductivity of the fluid, and $\gamma$ is the aligned angle. The boundary conditions for the problem are

$$
\begin{aligned}
& u=\varepsilon u_{w}, \quad v=v_{0}, T=T_{w}(x)=T_{\infty}+b x^{2} \text { at } y=0 \\
& u \rightarrow 0, T \rightarrow T_{\infty} \text { as } y \rightarrow \infty
\end{aligned}
$$

where $b$ is a some constant, and $v_{0}$ is the mass flux velocity. Introducing the stream function $\psi$ defined by $u=\partial \psi / \partial y$ and $v=-\partial \psi / \partial x$, equation (1) is satisfied identically. Then, Eqs. (2) and (3) can be written as 


$$
\begin{aligned}
\left(\frac{\partial \psi}{\partial y}\right)\left(\frac{\partial^{2} \psi}{\partial x \partial y}\right)-\left(\frac{\partial \psi}{\partial x}\right)\left(\frac{\partial^{2} \psi}{\partial y^{2}}\right) & \\
+\lambda\left[\left(\frac{\partial \psi}{\partial y}\right)^{2}\left(\frac{\partial^{3} \psi}{\partial y \partial x^{2}}\right)+\left(\frac{\partial \psi}{\partial x}\right)^{2}\left(\frac{\partial^{3} \psi}{\partial y^{3}}\right)\right. & \left.-2\left(\frac{\partial \psi}{\partial y}\right)\left(\frac{\partial \psi}{\partial x}\right)\left(\frac{\partial^{3} \psi}{\partial x \partial y^{2}}\right)\right] \\
& =v\left(\frac{\partial^{3} \psi}{\partial y^{3}}\right)-\frac{\sigma B_{0}^{2}}{\rho} \sin ^{2}(\gamma)\left(\frac{\partial \psi}{\partial y}\right) \\
\left(\frac{\partial \psi}{\partial y}\right)\left(\frac{\partial T}{\partial x}\right)-\left(\frac{\partial \psi}{\partial x}\right)\left(\frac{\partial T}{\partial y}\right) & =\frac{k}{\rho c_{p}}\left(\frac{\partial^{2} T}{\partial y^{2}}\right)
\end{aligned}
$$

Further, we seek for a similarity solution of Eqs. (5) and (6) subject to the boundary conditions (4) by introducing the following similarity transformation:

$$
\eta=\left(\frac{a}{v}\right)^{1 / 2} y, \quad \psi=(a v)^{1 / 2} x f(\eta), \quad \theta(\eta)=\frac{T-T_{\infty}}{T_{w}-T_{\infty}}
$$

where $\eta$ is the similarity variable, $f(\eta)$ is the dimensionless stream function, and $\theta(\eta)$ is the dimensionless variables for the temperature.

Then, by substituting similarity transformation (7) into Eqs. (5) and (6), we obtain the following nonlinear ordinary differential equations:

$$
\begin{gathered}
f^{\prime \prime \prime}+f f^{\prime \prime}-f^{\prime 2}-M \sin ^{2}(\gamma) f^{\prime}+\beta\left(2 f f f^{\prime \prime}-f^{2} f^{\prime \prime \prime}\right)=0 \\
\frac{1}{\operatorname{Pr}} \theta^{\prime \prime}+f \theta^{\prime}-2 f^{\prime} \theta=0
\end{gathered}
$$

and the boundary conditions (4) becomes

$$
\begin{aligned}
& f(0)=S, \quad f^{\prime}(0)=\varepsilon, \quad \theta(0)=1 \text { at } \eta=0 \\
& f^{\prime}(\eta) \rightarrow 0, \quad \theta(\eta) \rightarrow 0 \text { as } \eta \rightarrow \infty
\end{aligned}
$$

Here primes denote differentiation with respect to $\eta, \varepsilon$ is the stretching/shrinking parameter with $\varepsilon>0$ for stretching and $\varepsilon<0$ shrinking, $\beta$ is the dimensionless Maxwell parameter, $M$ is the magnetic parameter, $\operatorname{Pr}$ is the Prandtl number, and $S$ is the suction/injection parameter with $S>0$ for suction and $S<0$ for injection, which are defined as

$$
\beta=\lambda a, \quad M=\frac{\sigma B_{0}{ }^{2}}{a \rho}, \quad \operatorname{Pr}=\frac{\mu c_{p}}{k}, \quad S=-\frac{v_{0}}{\sqrt{a v}}
$$


The physical quantities of interest are the skin friction coefficient $C_{f}$ and the local Nusselt number $N u_{x}$ which is defined as

$$
C_{f}=\frac{\tau_{w}}{\rho u_{w}{ }^{2}(x) / 2}, \quad N u_{x}=\frac{x q_{w}}{k\left(T_{w}-T_{\infty}\right)}
$$

where $\tau_{w}=\mu(\partial u / \partial y)_{y=0}$ and $q_{w}=-k(\partial T / \partial y)_{y=0}$ are the shear stress, and heat flux at the surface, respectively. By using the similarity transformation (7), we obtain

$$
\frac{1}{2} C_{f} R e_{x}^{1 / 2}=f^{\prime \prime}(0), \quad N u_{x} / R e_{x}^{1 / 2}=-\theta^{\prime}(0)
$$

where $R e_{x}=u_{w}(x) x / v$ is the local Reynolds number based on the stretching velocity $u_{w}(x)=a x$ whereas the skin friction coefficient is represented by $f^{\prime \prime}(0)$ while the heat transfer coefficient is represented by $-\theta^{\prime}(0)$, respectively.

\section{Numerical solution}

The nonlinear equations (8) and (9) are transformed into a system of five first order differential equations as follows:

$$
\begin{aligned}
& \frac{d f_{0}}{d \eta}=f_{1}, \\
& \frac{d f_{1}}{d \eta}=f_{2}, \\
& \frac{d f_{2}}{d \eta}=\frac{\left(f_{1}\right)^{2}+M \sin ^{2}(\gamma) f_{1}-f_{0} f_{2}-2 \beta f_{0} f_{1} f_{2}}{1-\beta f_{0}^{2}}, \\
& \frac{d \theta_{0}}{d \eta}=\theta_{1}, \\
& \frac{d \theta_{1}}{d \eta}=\operatorname{Pr}\left[2 f_{1} \theta_{0}-f_{0} \theta_{1}\right]
\end{aligned}
$$

Subsequently the boundary conditions (10) take the form,

$$
\begin{aligned}
& f_{0}(0)=S, \quad f_{1}(0)=\varepsilon, \quad f_{1}(\infty)=0, \\
& \theta_{0}(0)=1, \theta_{0}(\infty)=0
\end{aligned}
$$

Here $f_{0}=f(\eta)$ and $\theta_{0}=\theta(\eta)$. The aforementioned boundary value problem is first converted into an initial value problem (IVP) by appropriately guessing the missing slopes $f_{2}(0)$ and $\theta_{1}(0)$. The resulting IVP is solved by shooting method for a set of parameters appearing in the governing equations with a known value of $f_{2}(0)$ and $\theta_{1}(0)$. The 
convergence criterion largely depends on fairly good guesses of the initial conditions in the shooting technique. The iterative process is terminated until the relative difference between the current iterative values of $f_{2}(0)$ matches with the previous iterative value of $f_{2}(0)$ up to a tolerance of $10^{-6}$.

\section{Results and discussions}

The governing partial differential equations of flow and heat transfer are transformed into a system of non-linear ordinary differential equations by using the similarity transformation. Eqs. (8) and (9) subjected to the boundary conditions (10) are solved numerically by using a shooting method. The accuracy of the above mentioned numerical method is validated by direct comparisons with earlier published results in some limiting cases which are presented in Table 1 and Table 2. It is noted that the present results are in good agreement with the existing results.

Table 1. Comparison of skin friction coefficient $f^{\prime \prime}(0)$ for different values of Maxwell parameter $\beta$ when $\operatorname{Pr}=1, M=0, S=0$ and $\varepsilon=1$ (stretching case).

\begin{tabular}{|c|c|c|c|c|}
\hline \multirow{2}{*}{$\beta$} & \multicolumn{4}{|c|}{$f^{\prime \prime}(0)$} \\
\cline { 2 - 5 } & Sadeghy et al. [22] & Mamaloukas et al. [29] & Abel et al. [19] & Present results \\
\hline 0 & -1.0000 & -0.999962 & -0.999962 & -1.00000005 \\
\hline 0.2 & -1.0549 & - & -1.051948 & -1.05188989 \\
\hline 0.4 & -1.10084 & -1.101850 & -1.101850 & -1.10190327 \\
\hline 0.6 & -1.0015016 & - & -1.150163 & -1.15013734 \\
\hline 0.8 & -1.19872 & -1.196692 & -1.196692 & -1.19671125 \\
\hline 1.2 & - & -1.285257 & -1.285257 & -1.28536326 \\
\hline
\end{tabular}

Table 2. Comparison of skin friction coefficient $f^{\prime \prime}(0)$ for different values of suction parameter $S$ when $\beta=0, \operatorname{Pr}=1, M=2$ and $\varepsilon=-1$ (shrinking case).

\begin{tabular}{|c|c|c|c|}
\hline \multirow{2}{*}{$S$} & \multicolumn{3}{|c|}{$f^{\prime \prime}(0)$} \\
\cline { 2 - 4 } & Muhaimin et al. [16] & Bhattacharyya [17] & Present results \\
\hline 2 & 2.414214 & 2.414300 & 2.41421357 \\
\hline 3 & 3.302776 & 3.302750 & 3.30277563 \\
\hline 4 & 4.236068 & 4.236099 & 4.23606797 \\
\hline
\end{tabular}

Figure 2 and 3 shows the effect of aligned angle and magnetic parameter on velocity profiles for the stretching case, respectively. It can be observed that the increase in aligned angle and magnetic parameter decreases the velocity profiles of the fluid for the stretching case. The reason behind this behavior is increased in aligned angle causes to strengthen the magnetic field. Due to enhanced magnetic field, it generates opposite force to the flow which is called Lorentz force. This force declines the velocity boundary layer thickness.

Figure 4 and 5 demonstrate the effect of the aligned angle on the velocity and temperature profiles for the shrinking case, respectively. It is observed that an increase in the aligned angle enhances the velocity profiles and depreciates the temperature profiles for the shrinking case. We observed that the Lorentz force helps to enhance the flow in shrinking case. 


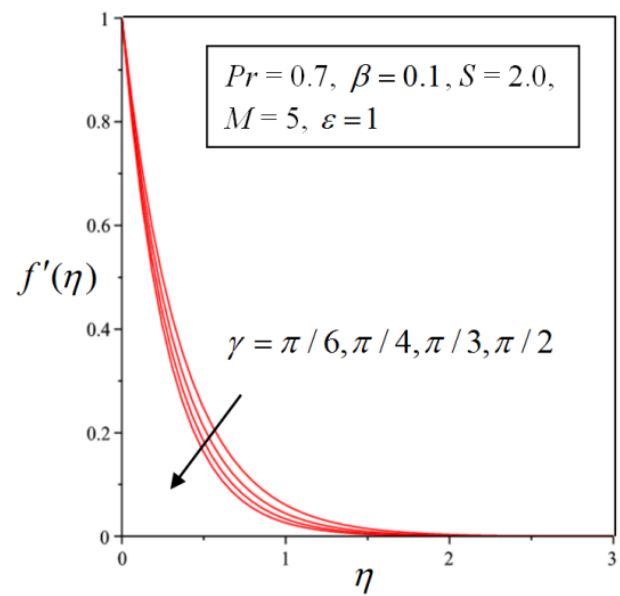

Fig. 2. Effect of the aligned angle $\gamma$ on the velocity profiles.

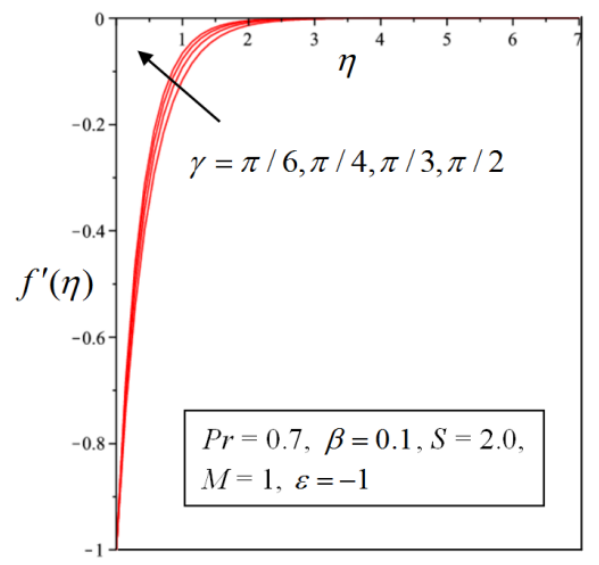

Fig. 4. Effect of the aligned angle $\gamma$ on the velocity profiles.

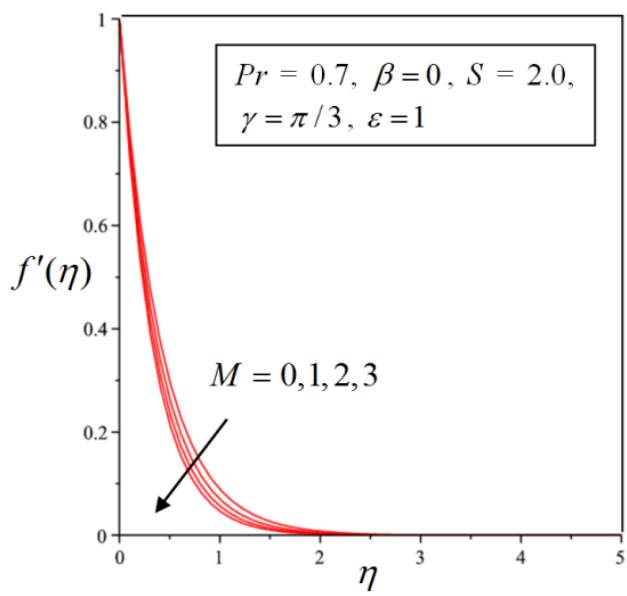

Fig. 3. Effect of the magnetic parameter $M$ on the velocity profiles.

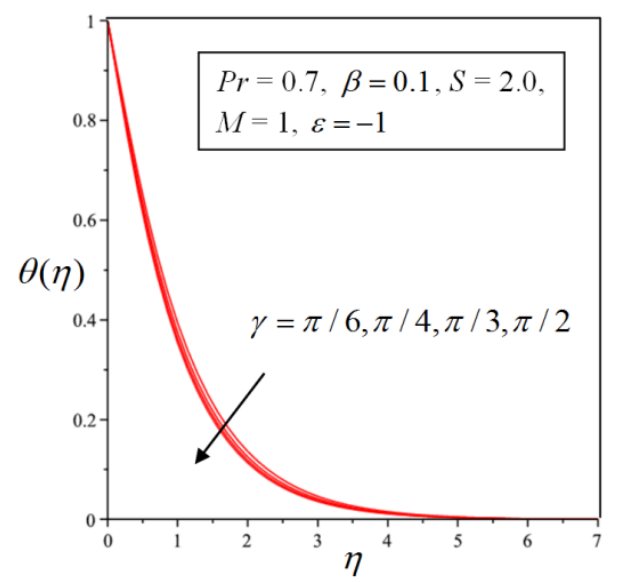

Fig. 5. Effect of the aligned angle $\gamma$ on the temperature profiles.

Meanwhile, Figure 6 and 7 are depicted to show the effect of magnetic parameter on velocity and temperature profiles in shrinking sheet. As expected, an increase in the magnetic parameter leads to increase the velocity and decrease the temperature of the fluid.

The effect of the Maxwell parameter on velocity and temperature profiles displayed in Figure 8 and 9. An increase in the Maxwell parameter is noticed to increase the fluid velocity and decrease the fluid temperature in shrinking sheet.

Figure 10 and 11 illustrate the effect of the aligned angle and magnetic parameter on the skin friction and heat transfer coefficients. It is found that the increasing of aligned angle and magnetic parameter has the capability to increase the value of the skin friction and heat transfer coefficients in shrinking sheet. This behavior also can be observed in Table 3. 


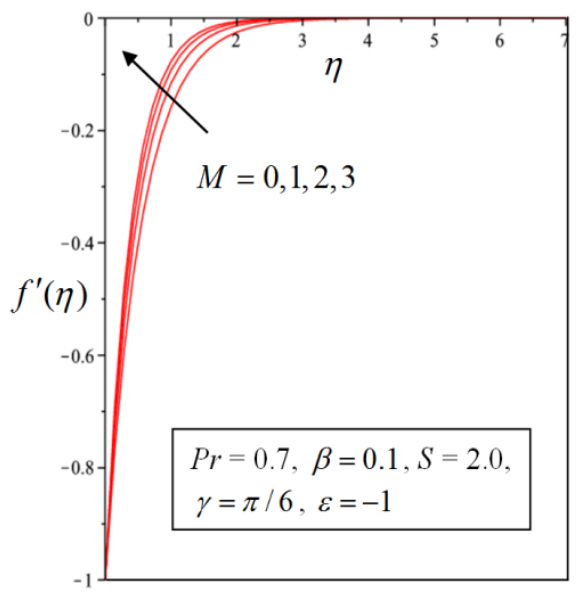

Fig. 6. Effect of the magnetic parameter $M$ on the velocity profiles.

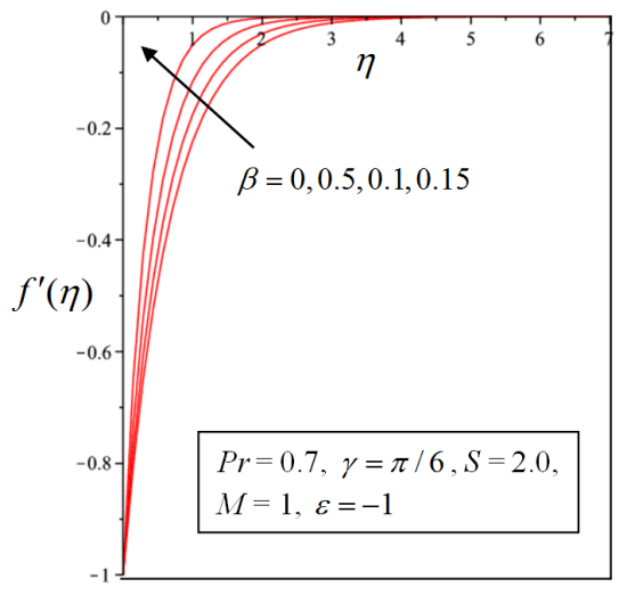

Fig. 8 Effect of the Maxwell parameter $\beta$ on the velocity profiles.

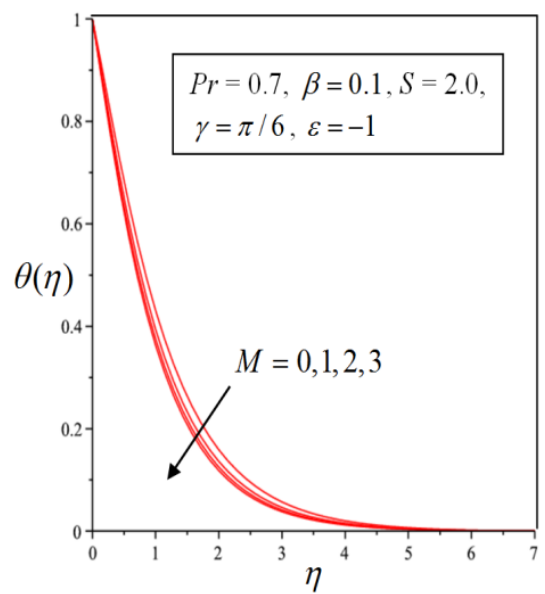

Fig. 7. Effect of the magnetic parameter $M$ on the temperature profiles.

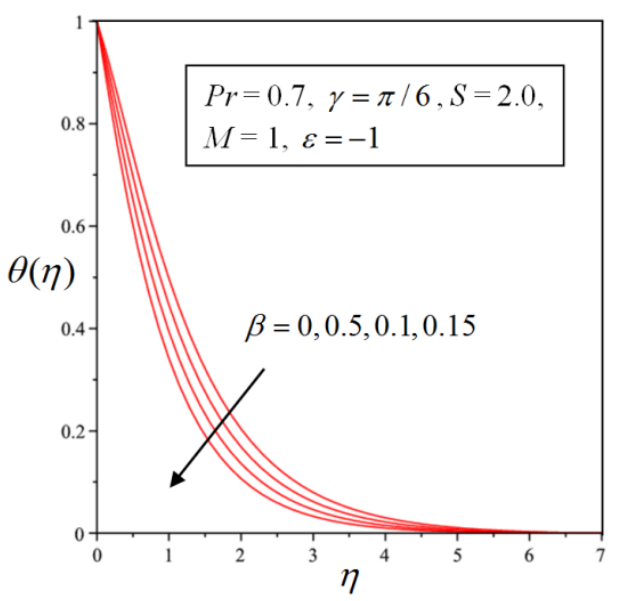

Fig. 9 Effect of the Maxwell parameter $\beta$ on the temperature profiles.

Table 3. Values of the skin friction $f^{\prime \prime}(0)$ and heat transfer $-\theta^{\prime}(0)$ coefficients for different values of aligned angle $\gamma$ and the magnetic parameter $M$ when $\operatorname{Pr}=0.7, \beta=0.1, S=2.0$, and $\varepsilon=-1$.

\begin{tabular}{|c|c|c|c|}
\hline$\gamma$ & $M$ & $f^{\prime \prime}(0)$ & $-\theta^{\prime}(0)$ \\
\hline \multirow{4}{*}{$\pi / 6$} & 0 & 1.81868494 & 0.59941014 \\
\cline { 2 - 4 } & 1 & 2.13643677 & 0.70462676 \\
\cline { 2 - 4 } & 2 & 2.36184718 & 0.76153317 \\
\cline { 2 - 4 } & 3 & 2.54657746 & 0.80073211 \\
\hline \multirow{4}{*}{$\pi / 4$} & 0 & 1.81866870 & 0.59795336 \\
\cline { 2 - 4 } & 1 & 2.36184718 & 0.76153317 \\
\cline { 2 - 4 } & 2 & 2.70691873 & 0.83005752 \\
\cline { 2 - 4 } & 3 & 2.98177503 & 0.87421087 \\
\hline
\end{tabular}




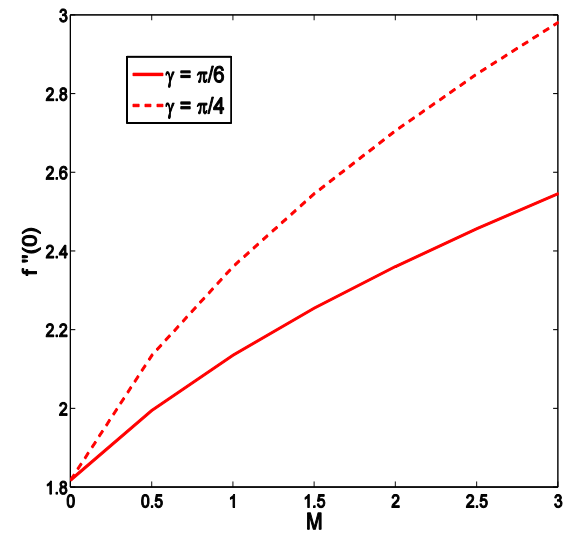

Fig. 10. Effect of the aligned angle and magnetic parameter on the skin friction coefficient when $\operatorname{Pr}=0.7, \beta=0.1, S=2.0$, and $\varepsilon=-1$.

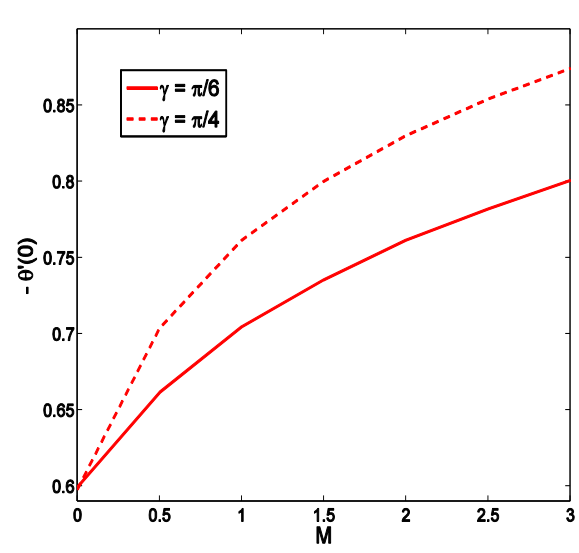

Fig. 11. Effect of the aligned angle and magnetic parameter on the heat transfer coefficient when $\operatorname{Pr}=0.7, \beta=0.1, S=2.0$, and $\varepsilon=-1$.

\section{Conclusion}

This paper presents a numerical solution for aligned magnetic field towards the flow and heat transfer of the upper-convected Maxwell (UCM) fluid over a stretching/shrinking sheet. By means of similarity transformation, the governing mathematical equations are reduced into ordinary differential equations which are then solved numerically using a shooting method. From the present investigation, the following conclusions can be drawn:

1. An increase in the aligned angle strengthens the magnetic field parameter and depreciates the velocity profiles in stretching case. However, increases in aligned angle enhances the fluid velocity for the shrinking case. Meanwhile, the fluid temperature decrease with the increase of aligned angle.

2. The increasing of magnetic parameter produces a decrease in the fluid velocity for the stretching case, but the opposite trend is observed in shrinking case. Besides, the fluid temperature decrease with the increase of magnetic parameter.

3. An increase in the Maxwell parameter is noticed to increase the fluid velocity and decrease the fluid temperature for the shrinking case.

4. The increasing of aligned angle and magnetic parameter has the capability to increase the value of the skin friction and heat transfer coefficients in shrinking sheet.

The authors would like to acknowledge Universiti Teknikal Malaysia Melaka (UTeM) for financial support under the UTeM short term grants PJP/2015/FTK(8C)/S01411 and PJP/2015/FTK(1C)/S01394.

\section{References}

1. B.C. Sakiadis, Am. Inst. Chem. Engineers J., 7, 26 (1961)

2. F. Tsou, E. Sparrow, R. Goldstein, Int. J. Heat Mass Transfer, 10, 219 (1967)

3. L.J. Crane, Z. Angew, Math. Phys., 21, 645 (1970) 
4. P.S. Gupta, A.S. Gupta. Can., J. Chem. Eng., 55, 744 (1977)

5. C.K. Chen, M. Char, Journal of Mathematical Analysis and Applications, 135, 568 (1988)

6. M.E. Ali, Warme Stoffubertragung, 29, 227 (1994)

7. M.E. Ali, International Journal of Heat and Fluid Flow, 16, 280 (1995)

8. R.S.R. Gorla, I. Sidawi, Appl. Sci. Res., 52, 247 (1994)

9. E.M.A. Elbashbeshy, J. Phys. D: Appl. Phys., 31, 1951 (1998)

10. T. Sarpkaya, AIChE J., 7, 324 (1961)

11. I.C. Liu, Int. Commun. Heat Mass Transfer, 32, 1075 (2005)

12. A. Chakrabarti, A.S. Gupta, Quart. Appl. Math. 37, 73 (1979)

13. T.C. Chiam, Int. J. Engng Sci., 33, 429 (1995)

14. S.P. Anjali Devi, M. Thiyagarajan, Heat and Mass Transfer, 42, 671 (2006)

15. T. Javed, Z. Abbas, M. Sajid, N. Ali, International Journal of Heat and Mass Transfer, 54, 2034 (2011)

16. Muhaimin, R. Kandasamy, Azme B. Khamis, Applied Mathematics and Mechanics, 1309 (2008)

17. K. Bhattacharyya, Chemical Engineering Research Bulletin, 12 (2011)

18. R.B. Bird, R.C. Armstrong, O. Hassager, Dynamics of polymeric liquids, 1, Wiley, New York (1987).

19. M.S. Abel, J.V. Tawade, M.M. Nandeppanavar, Meccanica, 47, 385 (2012)

20. M. Renardy, J. Non Newton, Fluid Mech., 68, 125 (1997)

21. K. Sadeghy, A.H. Najafi, M. Saffaripour, Int J Non-Linear Mech., 40, 1220 (2005)

22. K. Sadeghy, H. Hajibeygi, S.M. Taghavi, Int J Non-Linear Mech., 41, 1242 (2006)

23. T. Hayat, Z. Abbas, M. Sajid, Phys Lett A., 358, 396 (2006)

24. A. Alizadeh Pahlavan, V. Aliakbar, F. Vakili Farahani, K. Sadeghy, Commun Nonlinear Sci Numer Simul., 14, 473 (2009)

25. V. Aliakbar, A. Alizadeh Pahlavan, K. Sadeghy, Commun Nonlinear Sci Numer Simul., 14 (3), 779 (2009)

26. A. Alizadeh Pahlavan, K. Sadeghy, Commun Nonlinear Sci Numer Simul., 14 (4), 1355 (2009)

27. T. Hayat, Z. Abbas, M. Sajjid, Chaos Solitons Fractals., 39, 840 (2009)

28. B. Raftari, A. Yildirim, Computers and Mathematics with Applications, 59, 3328 (2010)

29. C. Mamaloukas, M.S. Abel, J.V. Tawade, U.S. Mahabaleswar, International Journal of Pure and Applied Mathematics, 66, 1 (2011)

30. C.S.K. Raju, N. Sandeep, C. Sulochana, V. Sugunamma, M. Jayachandra Babu, Journal of the Nigerian Mathematical Society, 34, 169 (2015)

31. C. Sulochana, N. Sandeep, V. Sugunamma, B. Rushi Kumar, Applied Nanoscience, 1 (2015) 